\title{
Force-induced diffusion in microrheology
}

\author{
Ch J Harrer ${ }^{1}$, D Winter ${ }^{2}$, J Horbach ${ }^{3}$, M Fuchs ${ }^{1}$ and Th Voigtmann ${ }^{1,4,5}$ \\ ${ }^{1}$ Fachbereich Physik, Universität Konstanz, 78457 Konstanz, Germany \\ 2 Institut für Physik, Johannes Gutenberg-Universität Mainz, Staudinger Weg 7, 55099 Mainz, Germany \\ ${ }^{3}$ Institut für Theoretische Physik II: Weiche Materie, Heinrich Heine-Universität Düsseldorf, \\ Universitätsstraße 1, 40225 Düsseldorf, Germany \\ ${ }^{4}$ Zukunftskolleg, Universität Konstanz, 78457 Konstanz, Germany \\ ${ }^{5}$ Institut für Materialphysik im Weltraum, Deutsches Zentrum für Luft- und Raumfahrt (DLR), \\ 51170 Köln, Germany \\ E-mail: thomas.voigtmann@uni-konstanz.de
}

\begin{abstract}
We investigate the force-induced diffusive motion of a tracer particle inside a glass-forming suspension when a strong external force is applied to the probe (active nonlinear microrheology). A schematic model of mode-coupling theory introduced recently is extended to describe the transient dynamics of the probe particle, and used to analyze recent molecular-dynamics simulation data. The model describes non-trivial transient displacements of the probe before a steady-state velocity is reached. The external force also induces diffusive motion in the direction perpendicular to its axis. We address the relation between the transverse diffusion coefficient $D_{\perp}$ and the force-dependent nonlinear friction coefficient $\zeta$. Non-diffusive fluctuations in the direction of the force are seen at long times in the MD simulation, while the model describes cross-over to long-time diffusion.
\end{abstract}

\section{Introduction}

In force-driven active microrheology, a probe particle that is embedded in a complex fluid is driven by an external force, and the resulting velocity of the probe is measured to assess the interactions between probe and host-liquid particles. This still quite novel technique can thus be used to investigate the local fluid properties [1]. If the chosen probe is similar in size to the host particles one thus gains access to a variety of local rheological properties of the system. This applies in particular to colloidal suspensions [2] or cellular fluids in biophysics [3].

In soft materials, small external forces are needed to drive the probe into the nonlinear-response regime. Then, the friction coefficient $\zeta\left(F_{\text {ex }}\right)$, defined through the average probe velocity $\langle\vec{v}\rangle$ in the steady-state limit as

$$
\zeta\left(F_{\mathrm{ex}}\right)\langle\vec{v}\rangle_{t \rightarrow \infty}=\vec{F}_{\mathrm{ex}},
$$

depends (usually nonlinearly) on the applied force $\vec{F}_{\text {ex }}$. The probe response mirrors the microscopic relaxation mechanisms at play in the host liquid. Understanding nonlinear active microrheology thus requires a thorough understanding of the probe-host interactions.

The nonlinear friction coefficient $\zeta\left(F_{\mathrm{ex}}\right)$ in viscoelastic fluids is seen to have three distinct regimes classified according to the strength of the external force. (i) At low forces, $F_{\mathrm{ex}} \rightarrow 0$, one recovers the linear response. Here, $\zeta$ is related to the probe-particle long-time self-diffusion coefficient via Einstein's relation, $D=k_{\mathrm{B}} T / \zeta$, where $k_{\mathrm{B}} T$ is the thermal energy. If the host liquid approaches a glass transition, $\zeta$ grows strongly in this regime (ignoring the possibility that small probes can in principle remain mobile even in the glass). In the ideal glass, $\zeta=\infty$. (ii) At intermediate forces, $F_{\mathrm{ex}} \approx F_{\mathrm{ex}}^{\mathrm{c}}$, a pronounced drop in $\zeta\left(F_{\mathrm{ex}}\right)$ is observed. This has been identified as the precursor of a non-equilibrium delocalization transition [4, 5]: even in an ideal glass, a forced probe can lead to a local melting of the glass, resulting in long-range motion of the probe. This is the regime we are most interested in. Notably, the threshold force required to delocalize the probe in the glass significantly exceeds typical thermal forces, $F_{\mathrm{ex}}^{\mathrm{c}} \gg k_{\mathrm{B}} T / \sigma$ (where $\sigma$ is a typical length characterizing static fluid-particle 
correlations, such as their size or average separation). (iii) At forces much higher than the threshold, a second plateau often is observed, i.e. $\zeta\left(F_{\mathrm{ex}} \rightarrow \infty\right)$ becomes independent of the force. This regime was first analyzed in dilute colloidal suspensions, where analytical calculations allowed $\zeta\left(F_{\text {ex }} \rightarrow\right.$ $0) / \zeta\left(F_{\text {ex }} \rightarrow \infty\right)=2$ to be established [6, 7]. This is not an effect of hydrodynamic interactions, as it has also been found in simulations of Brownian particles [4, 7]. Furthermore, $\zeta\left(F_{\text {ex }} \rightarrow \infty\right)>\zeta_{0}$ was found, where $\zeta_{0}$ is the solvent friction modeled in Brownian dynamics. This large-force plateau depends on the details of the microscopic dynamics and possibly on the driving mechanism; notably it is not found in recent simulations of a driven granular material [8].

In this contribution, we investigate the force-induced diffusion of the probe particle. Acting on the probe with a strong force causes not only a steady drift, but also random displacement fluctuations. The low-density limit in colloidal systems has been analyzed theoretically and with Brownian-dynamics simulations [9]. In the dense regime, recent molecular-dynamics (MD) simulations [10] have shown that the question of whether the random fluctuations become diffusive at long times is highly non-trivial. Moreover, the approach to the stationary limit, equation (1), is of interest, as it provides an experimental means to investigate the build-up and decay of transient force fluctuations in the system. Theoretical results on simplified models using random force fields [11] have revealed a rich scenario of classical diffusive, sub- and super-diffusive motion, tested in the mentioned simulation work.

To describe the nonlinear friction coefficient, an extension of the mode-coupling theory of the glass transition (MCT) was proposed [4]. In this theory, $\zeta\left(F_{\mathrm{ex}}\right)$ is given by a non-equilibrium Green-Kubo relation (generalized to strong external forcing), where transient force-force correlation functions appear. The latter are approximated in terms of the transient tracer density correlation functions and the equilibrium host-liquid density correlations. So far, dynamical quantities have only been calculated in a schematic version of the MCT model. Still, it has been possible to quantitatively explain the force-dependent friction values for various densities measured in simulation and experiment in all accessible regimes [5].

From the viewpoint of MCT it appears natural to quantify the dynamics beyond the single-time average appearing in equation (1) through the time-dependent density correlation functions. Both Brownian dynamics and schematic MCT show very similar correlators if one studies density fluctuations with wave numbers probing typical nearest-neighbor distances [4, 5]. Going beyond the local dynamics, recent molecular-dynamics computer simulations [10] investigated the mean-squared displacements (MSD) of the probe particle around the induced average motion. Two very interesting features were reported: first, in the direction perpendicular to the applied force, a diffusive MSD is found, indicating that the particle is delocalized not only in the direction of the force. Secondly, the MSD in the direction of the force shows superlinear growth over a number of decades in time, even after subtracting the obvious drift accounting for the finite average velocity.
We extend the schematic model of MCT proposed in [5] to include a description of the MSD in the two relevant directions. We use this model to give a quantitative fit of many of the quantities reported in [10], including the non-trivial time-dependent transient mean displacements of the particle before entering the steady state. The non-trivial super-diffusive power laws reported in [10] are not captured in the present MCT model, and we discuss possible reasons for this as well as possible ways to include them.

\section{Methods and techniques}

\subsection{Schematic mode-coupling theory: correlators}

We briefly summarize the equations defining the schematic MCT model of [5]. It consists of a correlator $\phi(t)$ that mimics the (equilibrium) density correlation functions of the host liquid. Its equation of motion reads

$$
\tau \partial_{t} \phi(t)+\phi(t)+\int_{0}^{t} m\left(t-t^{\prime}\right) \partial_{t^{\prime}} \phi\left(t^{\prime}\right) \mathrm{d} t^{\prime}=0
$$

$\tau$ is a parameter characterizing the collective short-time motion, and sets the unit of time in the model. The memory kernel $m(t)$ describes the slow structural relaxation close to the glass transition. Within MCT, it is expressed as a bilinear functional of the correlator $\phi$ itself; the simplest choice that recovers the asymptotic features predicted from the full MCT for typical hard-core interactions is [12]

$$
m(t)=v_{1} \phi(t)+v_{2} \phi(t)^{2},
$$

which is called the $\mathrm{F}_{12}$ model.

The parameters $\left(v_{1}, v_{2}\right)$ describe the equilibrium dynamics of the host liquid. In the model, a line of glass-transition points $\left(v_{1}^{c}, v_{2}^{c}\right)$ exists. There, the long-time limit $f=\lim _{t \rightarrow \infty} \phi(t)$ of the correlation function exhibits a discontinuous change: for small coupling coefficients, $f=0$ describes a liquid. For large couplings, the non-ergodicity parameter or glass form factor is positive, $f>0$, as the incomplete decay or the correlation function indicates an (amorphous) solid. Right at the transition, $f=f^{c}>0$ holds, and in this sense the glass transition is discontinuous. The specific point along the line of glass transitions determines the asymptotic shape of the correlation functions: in the liquid, these decay to and from an intermediate-time plateau given by $f^{c}$ by power laws $t^{-a}$ and $-t^{b}$, with non-universal exponents given by a parameter $\lambda$ that depends on the critical coupling coefficients. In the following numerical calculations, we fix $v_{2}^{c}=2$, corresponding to $v_{1}^{c}=2(\sqrt{2}-1)$, which results in $\lambda=1 / \sqrt{2} \approx 0.7$. This value is close to the one found from analyses of hard-sphere simulations [13]. It is customary to introduce a distance parameter $\varepsilon$ to the transition; we choose $\left(v_{1}, v_{2}\right)=\left(v_{1}^{c}, v_{2}^{c}\right)(1+\varepsilon)$ so that $\varepsilon>0$ signals a glass, $\varepsilon<0$ the liquid.

For the probe-particle correlation functions, two modes are introduced in the schematic model; one describing fluctuations in the direction of $\vec{F}_{\text {ex }}$ and one those perpendicular to 
it. In close analogy to equation $(2 a)$, we have

$$
\begin{gathered}
\zeta_{0}^{\mathrm{s}}\left(1-\mathrm{i} \kappa_{\|} F_{\mathrm{ex}}\right)^{-1} \partial_{t} \phi_{\|}^{\mathrm{s}}(t)+\phi_{\|}^{\mathrm{s}}(t) \\
+\int_{0}^{t} m_{\|}^{\mathrm{s}}\left(t-t^{\prime}\right) \partial_{t^{\prime}} \phi_{\|}^{\mathrm{s}}\left(t^{\prime}\right) \mathrm{d} t^{\prime}=0, \\
\zeta_{0}^{\mathrm{s}} \partial_{t} \phi_{\perp}^{\mathrm{s}}(t)+\phi_{\perp}^{\mathrm{s}}(t)+\int_{0}^{t} m_{\perp}^{\mathrm{s}}\left(t-t^{\prime}\right) \partial_{t^{\prime}} \phi_{\perp}^{\mathrm{s}}\left(t^{\prime}\right) \mathrm{d} t^{\prime}=0 .
\end{gathered}
$$

Here, $\zeta_{0}^{\mathrm{s}}$ sets the short-time relaxation for the probe particle in the absence of external forces. From the microscopic MCT [4], it is seen that the single-particle relaxation of density fluctuations corresponding to a wavevector $\vec{q}$ is modified by a term $\vec{q} \cdot\left(\vec{q} k_{\mathrm{B}} T-\mathrm{i} \vec{F}_{\text {ex }}\right)$. In the schematic model, where no explicit wavevector dependence is kept, this is replaced by the term $\left(1-\mathrm{i} \kappa_{\|} F_{\text {ex }}\right)$ appearing in equation ( $3 a$ ). The parameter $\kappa_{\|}$thus allows us to set a typical force scale relevant for the dynamics. Equation $(3 a)$ then already signals that the correlation function $\phi_{\|}^{\mathrm{s}}(t)$ becomes complex-valued, in stark contrast to equilibrium. This signals a force-induced shift of the average probe position in this direction. No such effect applies for density fluctuations with wavevectors obeying $\vec{q} \perp \vec{F}_{\text {ex }}$; their correlation functions remain real-valued, and are modeled in our schematic MCT by equation $(3 b)$. The memory kernels appearing in the above equations are, in the spirit of the mode-coupling approximation for tagged-particle density fluctuations [12] linear in $\phi(t)$ and linear in the $\phi_{\alpha}^{\mathrm{s}}(t)$ with $\alpha \in\{\|, \perp\}$. Importantly, the different directions are coupled. Specifically, we set [5]

$$
\begin{aligned}
& m_{\|}^{\mathrm{s}}(t)=\left(v_{1}^{\mathrm{s}} \phi_{\|}^{\mathrm{s}}(t)^{*}+v_{2}^{\mathrm{s}} \phi_{\perp}^{\mathrm{s}}(t)\right) \phi(t) /\left(1-\mathrm{i} \kappa_{\|} F_{\text {ex }}\right), \\
& m_{\perp}^{\mathrm{s}}(t)=\left(v_{1}^{\mathrm{s}} \phi_{\perp}^{\mathrm{s}}(t)+v_{2}^{\mathrm{s}} \operatorname{Re} \phi_{\|}^{\mathrm{s}}(t)\right) \phi(t) /\left(1+\left(\kappa_{\perp} F_{\mathrm{ex}}\right)^{2}\right) .
\end{aligned}
$$

The specific combination of the two probe correlators entering these memory kernels is justified by symmetry considerations of the full MCT [5]. It also ensures that in the schematic model the correlators obey the correct rules under $\vec{F}_{\text {ex }} \mapsto-\vec{F}_{\text {ex }}$ and that they embody the expected symmetry of the dynamics with respect to rotations around the force axis. Hence $F_{\text {ex }}$ enters quadratically in equation $(3 d)$; the parameter $\kappa_{\perp}$ allows us to fine-tune the force scale relevant for the motion perpendicular to $\vec{F}_{\text {ex }}$.

The coefficients $v_{1}^{\mathrm{s}}$ and $v_{2}^{\mathrm{s}}$ quantify the strength of the coupling between probe particle and host liquid. For $F_{\text {ex }}=0$, the model reduces to a single correlator $\phi_{\|}^{\mathrm{s}}(t)=\phi_{\perp}^{\mathrm{s}}(t)$ with a single coupling coefficient $v^{\mathrm{S}}=v_{1}^{\mathrm{S}}+v_{2}^{\mathrm{S}}$. This is the Sjoegren model [14] used successfully to describe incoherent scattering spectra of glass-forming liquids in equilibrium [12]. For arbitrary $F_{\mathrm{ex}}$, the model gives access to the nonlinear friction coefficient in terms of a schematic Green-Kubo relation [5]

$$
\zeta=\frac{\zeta_{0}^{\mathrm{s}}}{\kappa_{\|}}\left(1+\int_{0}^{\infty}\left(\mu_{\|} \operatorname{Re} \phi_{\|}^{\mathrm{s}}(t)+\mu_{\perp} \phi_{\perp}^{\mathrm{s}}(t)\right) \phi(t) \mathrm{d} t\right) .
$$

In the microscopic model, the Green-Kubo relation includes an integration over the space of wavevectors. In equation (4) it is assumed that the contributions coming from different spatial directions can be captured qualitatively by accounting for the two exemplary ones, $\alpha=\perp, \|$. The parameters $\mu_{\alpha}$ are introduced to adjust the relative weight of these qualitatively different contributions.

The model describes the force-induced delocalization of a probe particle in the ideal glass: there exists a critical force $F_{\mathrm{ex}}^{\mathrm{c}}$ below which the probe remains localized $(1 / \zeta=0)$, and above which long-range motion of the probe occurs ( $\zeta$ finite). In the tagged-particle correlation functions this is seen in the long-time limits $f_{\alpha}^{\mathrm{s}}=\lim _{t \rightarrow \infty} \phi_{\alpha}^{\mathrm{s}}(t)$. They remain finite for $F_{\mathrm{ex}}<F_{\mathrm{ex}}^{\mathrm{c}}$, but go to zero continuously as $F_{\mathrm{ex}} \rightarrow F_{\mathrm{ex}}^{\mathrm{c}}-0$, and remain zero for $F_{\mathrm{ex}}>F_{\mathrm{ex}}^{\mathrm{c}}$. In this sense, the schematic model used here describes the force-induced delocalization as a continuous transition. The asymptotic description of relaxation laws close to $F_{\mathrm{ex}}^{\mathrm{c}}$ has been worked out in detail [15].

The described generalization of schematic MCT to strongly driven probes contains the five (dimensionless) parameters corresponding to a time scale $\zeta_{0}^{\mathrm{s}}$, two force scales $\kappa_{\|}$and $\kappa_{\perp}$, and the fluid-probe coupling vertices $v_{1}^{\mathrm{s}}$ and $v_{2}^{\mathrm{s}}$. For given separation from the glass transition $\varepsilon$, it connects the change in the friction coefficient calculated from an approximated generalized Green-Kubo relation, equation (4), with the strength of the external force (given in $k_{\mathrm{B}} T / \sigma$ ). In the last equation, the non-Markovian contribution to the friction is approximated by the slow dynamics in the three correlators, the host liquid one, and the two tracer correlators describing the tracer fluctuations parallel and perpendicular to the force.

\subsection{Extension of the MCT model: displacements}

We now extend the above model in order to describe mean-squared displacements. These are obtained from the $\vec{q} \rightarrow 0$ limit of probe-particle density fluctuation correlations. The Appendix outlines the derivation in the microscopic MCT model. With the convention that the Cartesian z-direction coincides with the axis of $\vec{F}_{\text {ex }}$, we are dealing with three quantities: the averaged linear displacement along the force axis $\delta z(t)$, the MSD in this direction $\delta z^{2}(t)=\delta z^{2}(t)-$ $(\delta z(t))^{2}$, and the MSD in one of the orthogonal directions $\delta x^{2}(t)$. All lengths here are given relative to the characteristic fluid-particle scale $\sigma$. Schematically, the equations read

$$
\begin{aligned}
\zeta_{0}^{\mathrm{S}} \partial_{t} \delta z(t)+ & \int_{0}^{t} \hat{m}_{\|}^{\mathrm{s}}\left(t-t^{\prime}\right) \partial_{t^{\prime}} \delta z\left(t^{\prime}\right) \mathrm{d} t^{\prime}=\kappa_{\|} F_{\mathrm{ex}} \\
\zeta_{0}^{\mathrm{S}} \partial_{t} \delta z^{2}(t)+ & \int_{0}^{t} \hat{m}_{\|}^{\mathrm{s}}\left(t-t^{\prime}\right) \partial_{t^{\prime}} \delta z^{2}\left(t^{\prime}\right) \mathrm{d} t^{\prime}=2+2 \kappa_{\|} F_{\mathrm{ex}} \delta z(t) \\
& -2 \int_{0}^{t} K_{\|}^{\mathrm{s}}\left(t-t^{\prime}\right) \partial_{t^{\prime}} \delta z\left(t^{\prime}\right) \mathrm{d} t^{\prime} \\
\zeta_{0}^{\mathrm{S}} \partial_{t} \delta x^{2}(t)+ & \int_{0}^{t} \hat{m}_{\perp}^{\mathrm{s}}\left(t-t^{\prime}\right) \partial_{t^{\prime}} \delta x^{2}\left(t^{\prime}\right) \mathrm{d} t^{\prime}=2
\end{aligned}
$$

The memory kernels $\hat{m}_{\alpha}^{\mathrm{s}}(t)$ are obtained from longwavelength limits of the corresponding $m_{\alpha}^{\mathrm{s}}(t)$; lacking spatial information in the schematic model we set

$$
\begin{aligned}
& \hat{m}_{\|}^{\mathrm{s}}(t)=\zeta_{0}^{\mathrm{s}} \mu\left(\left(1-\mu_{\mathrm{rel}}\right) \operatorname{Re} \phi_{\|}^{\mathrm{s}}(t)+\mu_{\mathrm{rel}} \phi_{\perp}^{\mathrm{s}}(t)\right) \phi(t), \\
& \hat{m}_{\perp}^{\mathrm{s}}(t)=\zeta_{0}^{\mathrm{s}} \mu\left(\left(1-\mu_{\mathrm{rel}}^{\prime}\right) \operatorname{Re} \phi_{\|}^{\mathrm{s}}(t)+\mu_{\mathrm{rel}}^{\prime} \phi_{\perp}^{\mathrm{s}}(t)\right) \phi(t), \\
& K_{\|}^{\mathrm{s}}(t)=-\zeta_{0}^{\mathrm{s}} \mu \mu_{K} \operatorname{Im} \phi_{\|}^{\mathrm{s}}(t)
\end{aligned}
$$


where the four new parameters $\mu$ are dimensionless coupling parameters akin to the vertices $v_{1,2}^{\mathrm{s}}$.

Equation (5a) implies that the average probe motion follows the (projected as contained in $\kappa$ ) external force with a friction that contains an instantaneous solvent contribution and a non-Markovian one, arising from the tracer-fluid correlations. Only the temporal, not the spatial, correlations between probe and fluid are kept in the schematic model. Yet, the limit of vanishing wavevector is nonanalytic in the microscopic MCT, which implies that the $\vec{q} \rightarrow 0$ limits in the force kernel depend on direction (see the Appendix), and thus that $\mu_{\text {rel }}$ differs from $\mu_{\text {rel }}^{\prime}$. The friction felt by the probe decorrelates because of density fluctuations in the bath (modeled by $\phi(t)$ ) and parallel and transverse probe motion. Only the real part of the latter contributes because of the symmetry embedded in the microscopic equations. The MSD in the direction perpendicular to the applied force obeys an equation similar to the one in equilibrium, but the retarded friction kernel contains the force-induced probe fluctuations. The structural correlations in the force kernels are affected by the force also in the perpendicular direction, and thus the transverse MSD becomes force dependent. The mean-squared displacement in the direction of the applied force contains terms that correspond to the square of the average drift motion in the asymptotic long-time limit. For intermediate times, the time dependence of the kernels causes non-diffusive motion. Additionally, equation $(5 b)$ contains a memory kernel $K_{\|}^{\mathrm{s}}$ arising from force-stress correlations of the tracer. Little experience with this higher order correlation function exists. The choice in equation $(6 c)$ is suggested by numerical investigations of the full MCT; this term corresponds to the next-to-leading order in $q \rightarrow 0$.

In equilibrium $\left(F_{\mathrm{ex}}=0\right)$, equations (5) and (6) correctly recover that $\delta z(t) \equiv 0$, as there is no average motion of the tracer in any direction. The equations for $\delta x^{2}(t)=\delta z^{2}(t)$ in this case are the ones obtained earlier for the MSD of glass-forming systems [16], restoring spatial isotropy.

At long times, the equations can be analyzed in terms of their Laplace transforms. One has to distinguish two cases: if the density fluctuations of the host liquid and the probe are arrested (as is the case for low forces in the glass), the integrals over the memory kernels in equation (5) diverge, and one obtains finite linear and mean-squared displacements, indicating localization. We briefly quote the result for localized density fluctuations. The mean-squared displacement in the transverse direction in this case shows a long-time plateau related to the probe particle's localization length, $\delta x^{2}(t) \rightarrow 2 l_{\perp}^{2}$, with $l_{\perp}^{2}=1 / \hat{m}_{\|}^{\mathrm{s}}(t \rightarrow \infty)$. For the force direction, we obtain, under the assumption that also $K_{\|}^{\mathrm{s}}(t \rightarrow$ $\infty) \rightarrow$ const.,

$$
\begin{aligned}
\delta z(\infty) & =\frac{\kappa_{\|} F_{\mathrm{ex}}}{\hat{m}_{\|}^{\mathrm{s}}(\infty)}, \\
\delta \bar{z}^{2}(\infty) & =\frac{2+\left(\kappa_{\|} F_{\mathrm{ex}}\right)^{2} / \hat{m}_{\|}^{\mathrm{s}}(\infty)}{\hat{m}_{\|}^{\mathrm{s}}(\infty)}-\frac{2\left(\kappa_{\|} F_{\mathrm{ex}}\right) K_{\|}^{\mathrm{s}}(\infty)}{\hat{m}_{\|}^{\mathrm{s}}(\infty)^{2}} .
\end{aligned}
$$

Positivity of the result is recognized by recalling that due to equation $(6 c), K_{\|}^{\mathrm{s}}(t) \leq 0$ will typically hold.
Considering a liquid host, the decay of $\phi(t) \rightarrow 0$ as $t \rightarrow \infty$ ensures that the memory kernels remain finite. In this case, we obtain $\delta x^{2}(t) \sim 2 D_{\perp} t$ and $\delta z(t) \sim v t$ with

$$
\begin{gathered}
v=\frac{\kappa_{\|} F_{\mathrm{ex}}}{\zeta_{0}^{\mathrm{s}}+\int_{0}^{\infty} \hat{m}_{\|}^{\mathrm{s}}(t) \mathrm{d} t}, \\
D_{\perp}=\frac{1}{\zeta_{0}^{\mathrm{s}}+\int_{0}^{\infty} \hat{m}_{\perp}^{\mathrm{s}}(t) \mathrm{d} t^{\prime}} .
\end{gathered}
$$

From the steady-state velocity, the friction coefficient is also obtained:

$$
\zeta=F_{\mathrm{ex}} / v=\zeta_{0}^{\mathrm{s}} / \kappa_{\|}+\frac{1}{\kappa_{\|}} \int_{0}^{\infty} \hat{m}_{\|}^{\mathrm{s}}(t) \mathrm{d} t .
$$

Comparison with equation (4) shows that the schematic model is self-consistent regarding the two routes to calculate $\zeta$ (via the Green-Kubo relation and the Einstein relation), if we identify $\mu_{\|}=\mu\left(1-\mu_{\text {rel }}\right)$ and $\mu_{\perp}=\mu \mu_{\text {rel }}$.

To assess the long-time behavior of the nonaffine MSD in the force direction we consider the Laplace transform of equation $(5 b), \delta \hat{z}^{2}(s)=\int_{0}^{\infty} \mathrm{d} t \exp [-s t] \delta z^{2}(t)$,

$$
\begin{aligned}
\delta \hat{z}^{2}(s)= & \frac{2\left(\kappa_{\|} F_{\mathrm{ex}}\right)^{2}}{s^{3}\left(\zeta_{0}^{\mathrm{s}}+\hat{\hat{m}}_{\|}^{\mathrm{s}}(s)\right)^{2}} \\
& +\frac{2-2\left(\kappa_{\|} F_{\mathrm{ex}}\right) \hat{K}_{\|}^{\mathrm{s}}(s) /\left(\zeta_{0}^{\mathrm{s}}+\hat{\hat{m}}_{\|}^{\mathrm{s}}(s)\right)}{s^{2}\left(\zeta_{0}^{\mathrm{s}}+\hat{\hat{m}}_{\|}^{\mathrm{s}}(s)\right)} .
\end{aligned}
$$

The Abelian theorem ensures that the $t \rightarrow \infty$ limit of $\delta z^{2}(t)$ is given by the $s \rightarrow 0$ limit of this expression. Assuming that all memory terms appearing here are decaying functions so that their $s \rightarrow 0$ limits are finite, the leading term in equation (10) is the second one. It yields $\delta z^{2}(t) \sim v^{2} t^{2}$ and is identified as the affine drift contribution, i.e. it is asymptotically exactly canceled by $(\delta z(t))^{2}$. Hence, as long as $\hat{K}_{\|}^{\mathrm{s}}(s \rightarrow 0)$ remains regular, the dominant contribution to the true long-time limit in $\delta \bar{z}^{2}(t)=\delta z^{2}(t)-(\delta z(t))^{2}$ is diffusive in the model, $\delta \bar{z}^{2}(t) \sim$ $2 D_{\|} t$, with

$$
D_{\|}=\frac{1-\left(\kappa_{\|} F_{\mathrm{ex}}\right) \int_{0}^{\infty} K_{\|}^{\mathrm{s}}(t) \mathrm{d} t}{\zeta_{0}^{\mathrm{s}}+\int_{0}^{\infty} \hat{m}_{\|}^{\mathrm{s}}(t) \mathrm{d} t}+\frac{\left(\kappa_{\|} F_{\mathrm{ex}}\right)^{2} \int_{0}^{\infty} \hat{m}_{\|}^{\mathrm{s}}(t) t \mathrm{~d} t}{\left(\zeta_{0}^{\mathrm{s}}+\int_{0}^{\infty} \hat{m}_{\|}^{\mathrm{s}}(t) \mathrm{d} t\right)^{3}}
$$

Intriguingly, the appearance of the different memory kernels in equations (9) causes non-trivial Einstein relations in the different spatial directions. Moreover, the Markovian limit (to replace the time-dependent friction kernels with their integrated strength at long times, as familiar from hydrodynamics) may hold in quite different time windows, opening a window of non-diffusive intermediate dynamics.

\subsection{Molecular-dynamics computer simulation}

Simulations are performed on a glass-forming binary Yukawa fluid as described earlier [10]. The two particle types are referred to as $\mathrm{A}$ and $\mathrm{B}$. The pair interaction potential between a particle of type $\alpha$ and one of type $\beta$ reads $V_{\alpha \beta}(r)=$ $\epsilon_{\alpha \beta} \sigma_{\alpha \beta} \exp \left[-k\left(r-\sigma_{\alpha \beta}\right)\right] / r$, where $r$ is the interparticle 
distance. The $\sigma_{\alpha \beta}$ are the interaction diameters and chosen as $\sigma_{\mathrm{AA}}=\sigma=1$ (setting the unit of length in the simulation), $\sigma_{\mathrm{AB}}=1.1 \sigma$, and $\sigma_{\mathrm{BB}}=1.2 \sigma$. The energy scale is fixed by $\epsilon_{\mathrm{AA}}=\epsilon=1$, and the remaining parameters are $\epsilon_{\mathrm{AB}}=1.4 \epsilon$ and $\epsilon_{\mathrm{BB}}=2 \epsilon$. The screening parameter is set to $k=6 / \sigma$. The particle species have equal masses, $m_{\mathrm{A}}=m_{\mathrm{B}}=m=1.0$. The potential is truncated at a distance where $V_{\alpha \beta}=10^{-7} \epsilon$.

The number density of the system is fixed at $\rho=$ $0.68 / \sigma^{3}$. An asymptotic analysis of the simulation data suggests $T_{\mathrm{c}} \approx 0.14$ to be the critical (idealized-glass transition) temperature of MCT. Two geometries are used in the simulation, both with periodic boundary conditions in all directions: for smaller forces, where the probe motion is slow enough, a cubic box with length $L=13.3 \sigma$ containing $N=1600$ particles is used. At large forces, it becomes necessary to avoid finite-size effects by elongating the box in the force direction by a factor of 8. All simulations start from well-equilibrated initial configurations. To gather statistics, the production runs are repeated for 1000 randomly selected probe particles, with lengths between $10^{5}$ and $3 \times 10^{6}$ simulation time steps.

To keep the temperature constant in the non-equilibrium simulation, a thermostat has to be used. We employ a dissipative particle dynamics (DPD) thermostat using the algorithm proposed by Peters ('Scheme II') [17] to integrate the equations of motion with a time step $\delta t=$ $0.0083 \sigma(m / \epsilon)^{1 / 2}$. The thermostat introduces dissipative forces proportional to the relative velocity between pairs of neighboring particles, which ensures conservation of local momentum and Galilean invariance. The friction $\zeta$ that controls the strength of the dissipative forces is related to the strength $\alpha$ of the thermal random forces of the DPD equations by the fluctuation-dissipation theorem, $\alpha^{2}=2 \zeta k T$. We choose $\zeta=12$ (for this choice, the dynamics is almost identical to that of a microcanonical MD simulation in the absence of external fields). For the case of shear-rate driven macrorheology, it has been verified that the results do not qualitatively depend on $\zeta$ [18]. Further details on the implementation of the DPD thermostat can be found in $[18,19]$. Note that no signs of crystallization or phase separation are found during the length of the simulation runs.

\section{Results}

We describe fits of the MD simulation data using the schematic model of MCT. The fit proceeds by first finding the best possible description of the equilibrium MSD for a given temperature. This fixes the parameter $\varepsilon, v^{\mathrm{s}}, \mu$ as well as $\tau=$ $\zeta_{0}^{\mathrm{S}}$. There is some ambiguity since the short-time dynamics of our model is Brownian $\left(\delta x^{2} \sim 2 k_{\mathrm{B}} T t / \zeta_{0}^{\mathrm{s}}\right.$ for $\left.t \rightarrow 0\right)$, while that of the MD simulations is Newtonian $\left(\delta x^{2} \sim k_{\mathrm{B}} T / m t^{2}\right.$ for $t \rightarrow 0$ ). To accommodate the natural shift of the time scale in the MD simulations, where temperature is the control parameter that is varied, we set $\tau=1 / \sqrt{T}$ in the calculations. Then, the fit is optimized to yield a reasonable description of both the friction coefficient and the averaged displacements along the force direction, for all forces available, and at each temperature. Finally, the agreement with the MSD in

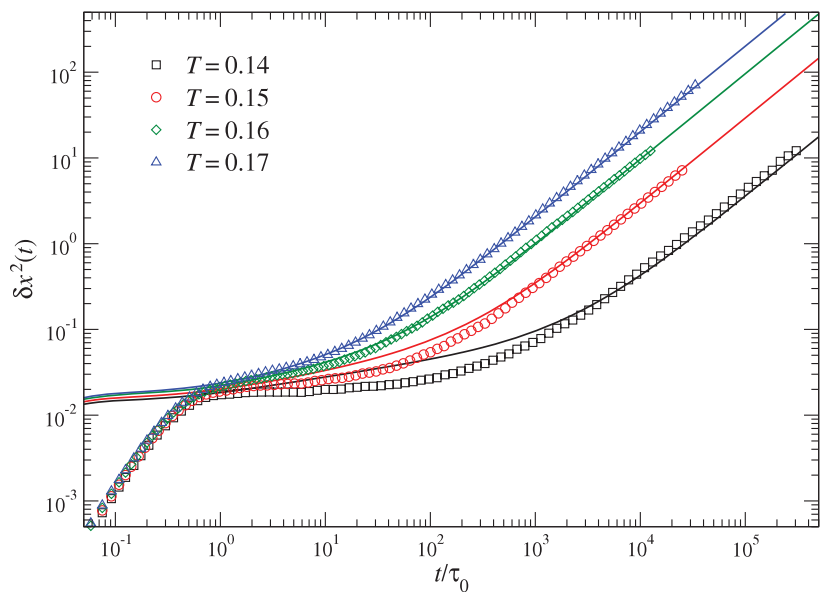

Figure 1. Equilibrium mean-squared displacements from molecular-dynamics simulation (symbols for different temperatures as labeled). Lines are fits using the schematic model.

the perpendicular direction, and the corresponding diffusion coefficient is checked.

To simplify the procedure, we try to keep as many parameters fixed as possible. In particular, the parameters $\kappa_{\perp}=2.25$ and $\kappa_{\|}=4.5$ are kept fixed. Here and in the following we use MD simulation units, where energy and length units are given by the potential parameters, unless otherwise noted. Since lengths appearing in the schematic model have been non-dimensionalized by some characteristic scale like $\sigma$, comparison to the MD data requires conversion to simulation units. In the present fits, this additional freedom was not used, and it was assumed that the schematic model gives lengths in units of the Yukawa MD parameters.

We first discuss the equilibrium MSD, shown in figure 1. The MD simulations display the usual quiescent glass-transition scenario: initially, features of the short-time single-particle dynamics are seen; ballistic motion $\delta x^{2}(t) \sim$ $t^{2}$ in the simulation, while the model becomes diffusive at short times. The short-time regime is followed by a plateau whose length in time increases as temperature is lowered, and whose height corresponds to a fraction of the particle diameter-associated with Lindemann's criterion for the typical size of nearest-neighbor cages in dense systems with hard-core interactions [12]. At long times, diffusive motion sets in corresponding to a diffusion coefficient $D$ that drops with lowering temperature. The power-law extrapolation of $D \rightarrow 0$ allows us to estimate a critical temperature $T_{\mathrm{c}}$ that is identified as the MCT glass-transition temperature [20]. An asymptotic analysis of the simulation data yields $T_{\mathrm{c}} \approx$ 0.14 [10].

Our fits to the equilibrium MSD (lines in figure 1) capture these qualitative features, although the quantitative description is not accurate. To a large part, this stems from our use of diffusive instead of ballistic short-time dynamics. Still the fits serve to fix the parameters of the $F_{12}$ model, in particular $\varepsilon$. All parameters of the schematic-model fit are shown in table 1 . From the fitted relation $T(\varepsilon)$ one estimates the critical temperature $T_{\mathrm{c}}=T(\varepsilon=0) \approx 0.135$, in $\operatorname{good}$ agreement with the asymptotic analysis quoted above. 


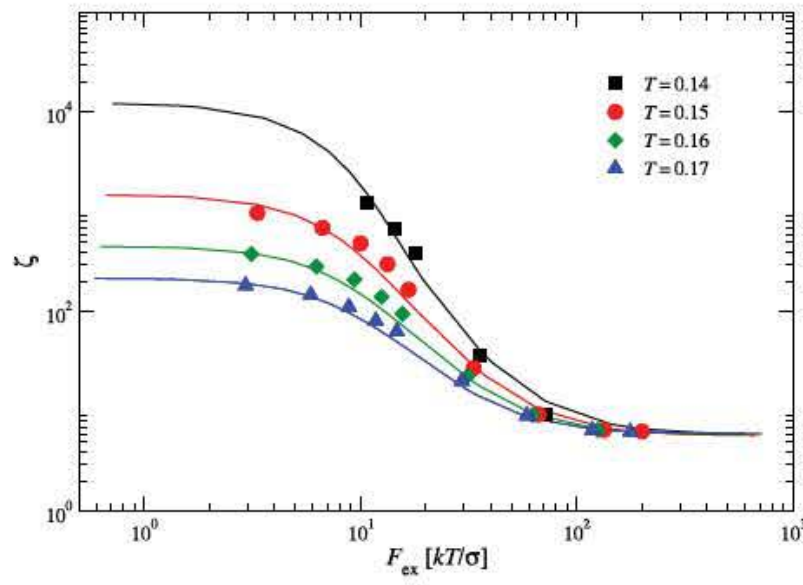

Figure 2. Friction coefficient $\zeta\left(F_{\text {ex }}\right)$ as a function of applied force $F_{\mathrm{ex}}$. Forces are measured in thermal units, $k_{\mathrm{B}} T / \sigma$, while $\zeta$ is given in simulation units. Symbols are results from MD simulation [10] for temperatures $T$ as labeled. Lines are fits using the schematic model discussed in the text.

Table 1. Fit parameters used in the fits of the schematic MCT model to the MD simulation data. Parameters not mentioned in the table are kept fixed for all temperatures: $\kappa_{\|}=4.5, \kappa_{\perp}=2.25$, $\mu_{\mathrm{rel}}^{\prime}=0$. The short-time scale varies as $\tau=\zeta_{0}^{\mathrm{s}}=1 / \sqrt{T}$.

\begin{tabular}{lccccc}
\hline$T$ & $\varepsilon$ & $v^{\mathrm{s}}$ & $\zeta_{0}^{\mathrm{s}} \mu$ & $\mu_{\text {rel }}$ & $\zeta_{0}^{\mathrm{s}} \mu_{K}$ \\
\hline 0.14 & -0.08 & 35 & 140 & 0.09 & 5.0 \\
0.15 & -0.20 & 32 & 130 & 0.10 & 2.5 \\
0.16 & -0.34 & 30 & 120 & 0.12 & 1.0 \\
0.17 & -0.48 & 28 & 115 & 0.14 & 0.5 \\
\hline
\end{tabular}

Figure 2 displays the probe-particle friction coefficient $\zeta\left(F_{\text {ex }}\right)$ for various temperatures close to the glass transition. The MD simulation results published recently [10] are shown as symbols. Forces have been converted from simulation units to thermal ones, $k_{\mathrm{B}} T / \sigma$, in order to ease comparison with previous analyses of microrheology in glass-forming systems. There it was found [4] that the threshold force for delocalization is of the order of $F_{\mathrm{ex}}^{\mathrm{c}} \approx 50 k_{\mathrm{B}} T / \sigma$. Estimating $F_{\mathrm{ex}}^{\mathrm{c}}$ by the turning point in the $\log \zeta$ versus $\log F_{\mathrm{ex}}$ curves in figure 2, this result also applies to the present Yukawa mixture.

Lines in figure 2 display our schematic-model fits. The fit quality is comparable to that of earlier fits to Brownian-dynamics data and experiment using the same model [5]. The critical force calculated within the MCT model, using the parameters listed in table $1, F_{\mathrm{ex}}^{\mathrm{c}} \approx$ $13.5 k_{\mathrm{B}} T / \sigma$, is in qualitative agreement with the estimate above. There $F_{\mathrm{ex}}^{\mathrm{c}} \gg k_{\mathrm{B}} T / \sigma$ holds, but the precise numerical value strongly depends on the probe-host interactions, as expected from the microscopic theory [4].

More information on the probe dynamics is given by its time-dependent response. Recall that the friction coefficient is defined only in the steady-state limit, were the particle undergoes a displacement in the force direction that is linear in time, $\delta z(t) \sim F_{\mathrm{ex}} t / \zeta\left(F_{\mathrm{ex}}\right)$. In figure 3 we show the transient linear displacement $\delta z(t)$ for times $t$ since the external force was applied, for two exemplary temperatures and various

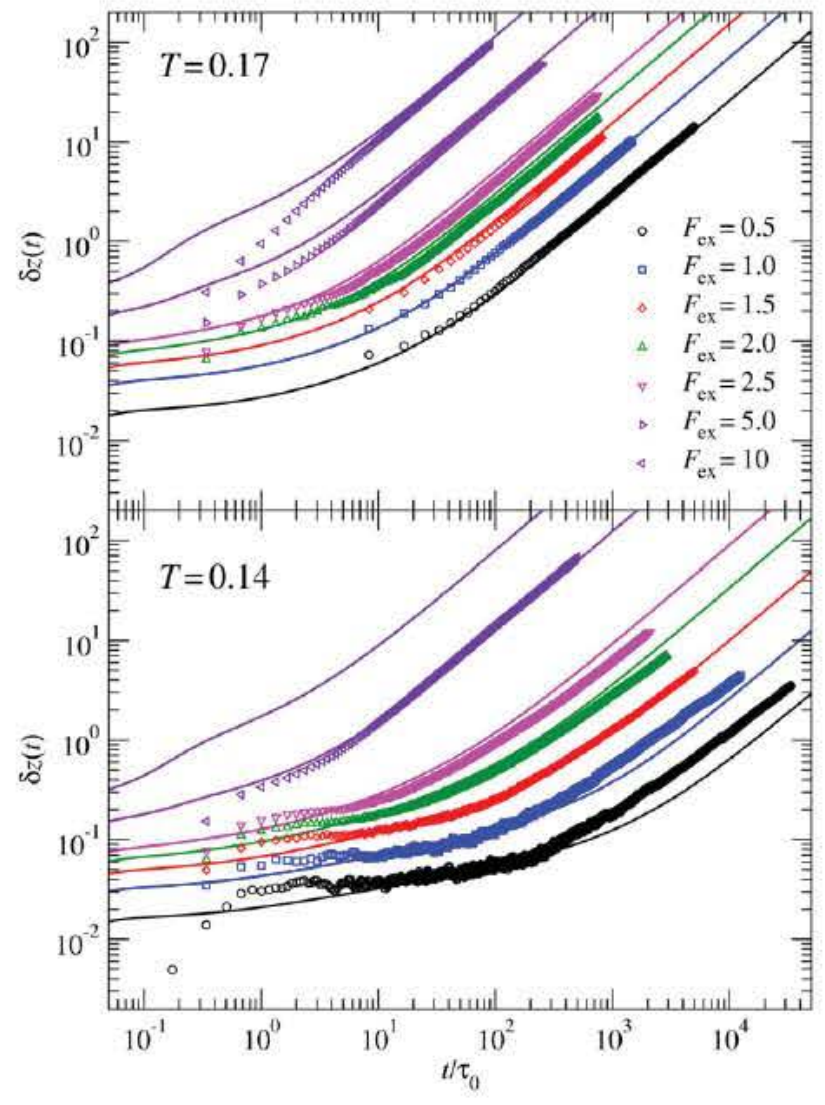

Figure 3. Average linear displacements $\delta z(t)$ for the microrheology MD simulations at temperatures $T=0.17$ (top) and $T=0.14$ (bottom). Symbols are simulation data for the external forces indicated in the legend in MD units; lines are fits with the schematic model.

external forces. As expected for a system showing slow dynamics, the time taken until a steady state is reached depends sensitively on the control parameters and grows as the glass transition is approached. This is exemplified by the $F_{\mathrm{ex}}=0.5$ curves in figure 3 . While for $T=0.17$, times $t \gtrsim$ $100 \tau_{0}$ are sufficient to reach the limit $\delta z(t) \sim t$, for $T=0.14 \mathrm{a}$ roughly ten times larger time, $t \gtrsim 1000 \tau_{0} /$ has to be reached. As the external force is increased, this time drops in line with the reduction of the nonlinear friction coefficient $\zeta\left(F_{\mathrm{ex}}\right)$ displayed in figure 2 .

A striking feature of the curves is that they show an intermediate force-dependent plateau. The height of this plateau depends roughly linearly on $F_{\mathrm{ex}}$, i.e. $\delta z(t) / F_{\mathrm{ex}} \sim$ const. holds at times $t \approx \tau_{0}$. This is qualitatively explained by the schematic model, as the fits shown in figure 3 demonstrate.

To understand this finding, recall that at intermediate times, we expect all correlation functions to be close to a plateau, which in the ideal glass is given by the glass form factors $f$ and $f_{\alpha}^{\mathrm{s}}$. Assuming that these terms are the subdominant force variation, equation (7) explains a linear increase of the intermediate plateau with $F_{\mathrm{ex}}$. In the double limit of approaching the probe-delocalization transition and the glass transition, one obtains a linear decrease of $f_{\alpha}^{\mathrm{s}}$, so that the plateau in $\delta z(t)$ should increase more strongly than linear upon approaching $F_{\mathrm{ex}}^{\mathrm{c}}$ from below. However, in the simulation 


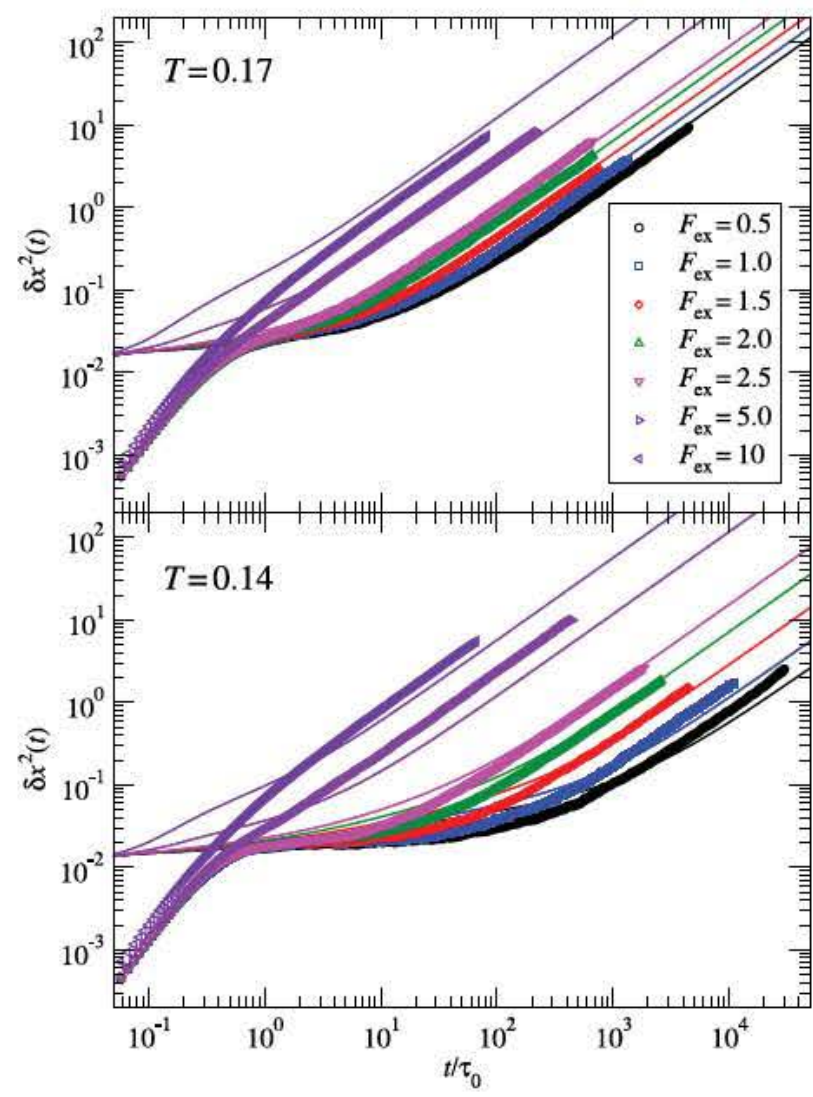

Figure 4. Mean-squared displacements in the direction perpendicular to the applied force, $\delta x^{2}(t)$, for temperatures $T=0.17$ (top) and $T=0.14$ (bottom) and external forces as labeled. Symbols are results of the MD simulation while lines correspond to the fits of the schematic model.

data, this effect is not yet seen, since the asymptotic analysis is valid only in a small region around the glass transition, and at $\varepsilon<0$ the final relaxation of the host-liquid correlation function determines the long-time behavior [15].

We now address the intriguing finding of [10], that an applied external force in the $z$-direction also induces fast diffusion in the perpendicular directions. This is visible in the mean-squared displacement in the $x$-direction, $\delta x^{2}(t)$, shown in figure 4. Note that the simulation data correspond to the stationary MSD, i.e. the one measured once the probe displacement $\delta z(t)$ has become linear in time. In principle, the theory rests upon calculating transient correlation functions, i.e. the schematic-model MSD is the one where $t=0$ corresponds to the time where the force is switched on. On the level of our comparison, we can neglect this difference; in particular both MSDs will agree with each other at long times [21].

The MSD show the same qualitative features as the equilibrium ones, but the long-time diffusion is governed by $F_{\text {ex }}$. This is clear from equation $(5 b)$, where the contribution from the memory kernel is decreased with increasing external force. As the fits (lines in figure 4) document, our model captures this force-induced acceleration of the diffusion in the transverse direction found in the MD simulations.

The enhancement of the orthogonal diffusion is clearly seen in a plot of $D_{\perp}$ as a function of $F_{\text {ex }}$, figure 5. This

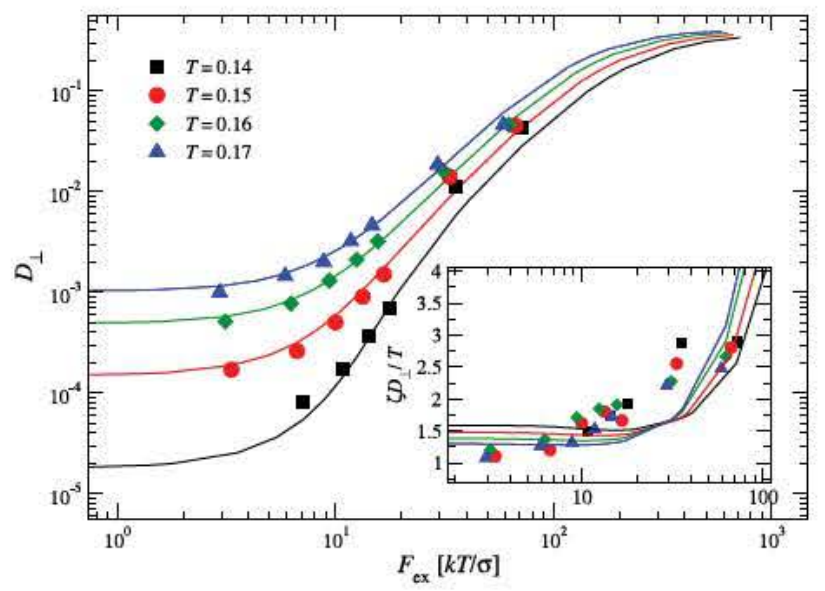

Figure 5. Diffusion coefficient $D_{\perp}$ in the direction perpendicular to $\vec{F}_{\text {ex }}$, as a function of the applied force $F_{\text {ex }}$, for temperatures as labeled. Symbols are MD simulation data while lines are fits from the schematic model. Inset: $\zeta \cdot D_{\perp} / T$ as a function of the applied force.

quantity displays the same qualitative behavior as $1 / \zeta\left(F_{\mathrm{ex}}\right)$, i.e. the mobility of the probe particle increases strongly in the regime around $F_{\mathrm{ex}} \approx F_{\mathrm{ex}}^{\mathrm{c}}$, and approaches a large-force plateau as $F_{\text {ex }} \rightarrow \infty$. From the equations of the schematic model, the analogy between $k_{\mathrm{B}} T / \zeta\left(F_{\mathrm{ex}}\right)$ and $D_{\perp}$ is clear from equations $(9 b)$ and $(9 c)$. At least asymptotically, the two memory kernels appearing there are closely linked, since all correlation functions that enter become proportional to each other [15]. As long as the memory-kernel contributions dominate, $D_{\perp} \zeta$ will thus asymptotically approach a constant close to the glass transition. Pre-asymptotic corrections and the regular variations in the parameters cause the product $\zeta \cdot D_{\perp} / T$ to vary. This is shown in the inset of figure 5 for both the simulation data and the schematic model.

The most striking result of the MD simulations [10] is that the MSD in the direction of the external force, corrected for the drift, remains super-diffusive. This is seen in figure 6, where $\delta \bar{z}^{2}(t)$ is shown. At long times and close to $T_{\mathrm{c}}$, the curves show power-law growth with an exponent close to 1.5 . Within the time window accessible in the simulations, they do not show a cross-over to long-time diffusion at $T=0.14$. At $T=0.17$, such a cross-over is eventually seen.

The schematic model we employ here is not able to reproduce the super-diffusive growth in $\delta \bar{z}^{2}(t)=\delta z^{2}(t)-$ $(\delta z(t))^{2}$. Note that the fits discussed so far fix all the parameters appearing in the model, except one additional memory kernel $K^{\mathrm{s}}(t)$ that appears in equation $(5 b)$, which only enters in the MSD in the direction of the force. Microscopically, this kernel is determined from a higher order contribution in wave number $q$. For the calculations presented in figure 6 as solid lines, we have fixed $K^{\mathrm{s}}(t)$ as outlined in section 2.1, i.e. proportional to $\operatorname{Im} \phi_{\|}^{\mathrm{s}}(t)$. In the schematic model, all correlation functions show exponential decay ultimately, if one considers control parameters that do not correspond to the critical delocalization point $F_{\mathrm{ex}}=F_{\mathrm{ex}}^{\mathrm{c}}$. Consequently, all memory integrals appearing in equation $(5 b)$ can be treated as Markovian on time scales long compared with this final relaxation. This leads to the 


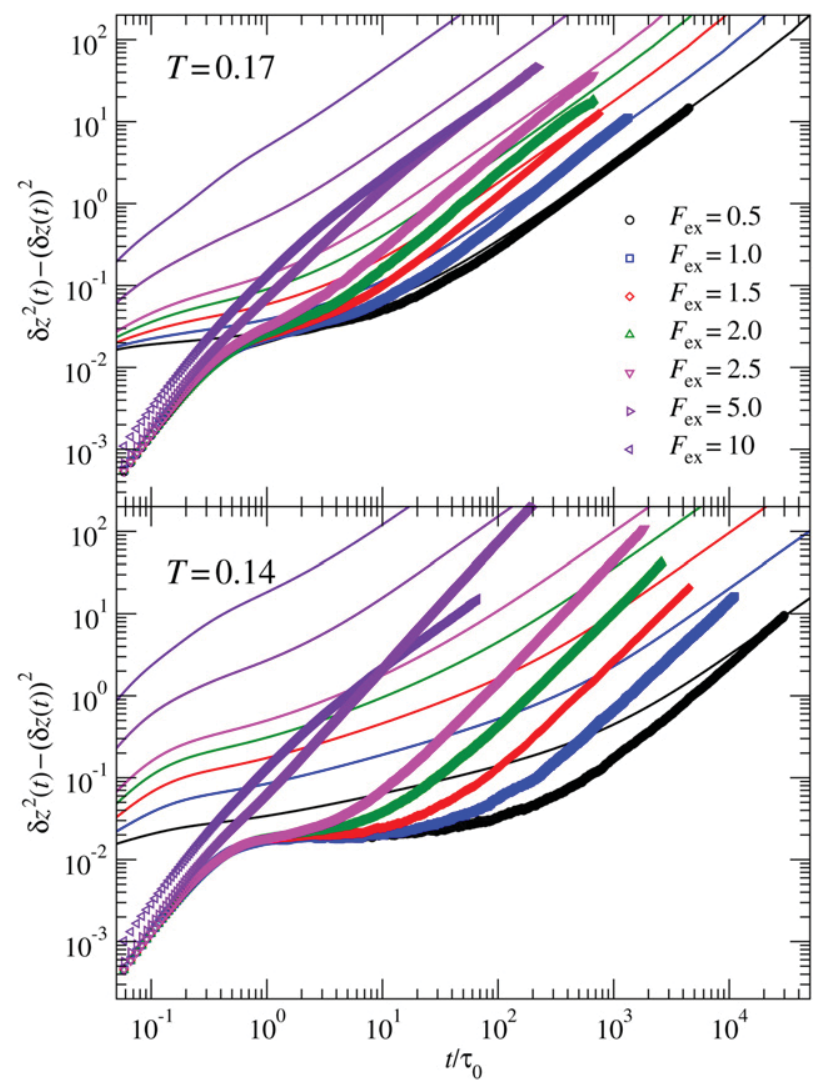

Figure 6. Mean-squared displacement, $\delta z^{2}(t)-(\delta z(t))^{2}$, in the direction of the applied force $\vec{F}_{\text {ex }}$, for $T=0.17$ (top) and $T=0.14$ (bottom) and forces as labeled. Symbols are results from MD simulations. Lines are the results within the schematic model evaluated for the parameters determined from the fits to the linear displacement $\delta z(t)$.

prediction of equation (11), i.e. $\delta \bar{z}^{2}(t)$, to become linear in time. For not too low temperature, $T=0.17$, and not too high force, this linear slope agrees with the one found in simulation. At high forces, the model fails to describe $D_{\|}$quantitatively; this is the regime where the short-time dynamics remains important.

Although the schematic model yields sub-diffusive $\delta \bar{z}^{2}(t)$ for our choice of parameters, super-diffusive behavior is not ruled out a priori in equation (5b). However, a better understanding of the role played by the new memory kernel $K_{\|}^{\mathrm{s}}(t)$ is required to assess the qualitative features of the solution to equation $(5 b)$.

Another qualitative difference between the forcedirection MSD in the simulation and in the schematic model is seen in figure 6 . The MD data at $T=0.14$ show an intermediate-time plateau that is roughly force independent, and agrees with the equilibrium Lindemann length. In contrast, the schematic model, equation (8), suggests an intermediate plateau whose value increases asymptotically proportional to $F_{\mathrm{ex}}^{2}$, if the force-induced change in the glass form factors are not yet the dominant contribution. Interestingly, one can read off the values reached in $\delta \bar{z}^{2}(t)$ at the onset of long-time diffusion from the MD data for $T=0.17$. These values, to within the uncertainty of their determination, strongly increase with $F_{\mathrm{ex}}$ (where $F_{\mathrm{ex}}^{2}$ is the obvious contribution that can arise respecting symmetries).

\section{Conclusions}

We have demonstrated that a simple three-correlator schematic model of MCT is able to quantitatively account for several quantities evaluated in recent MD simulations of force-driven active nonlinear microrheology. The fits of the schematic model follow the same spirit as earlier ones to friction coefficients obtained from Brownian-dynamics simulation and colloidal experiment. They extend them by addressing time-dependent quantities characterizing the probe-particle motion outside the steady-state regime.

Specifically, we have addressed the force-induced longrange motion of the probe particle in a host liquid close to its glass transition. Application of a constant external force $\vec{F}_{\text {ex }}$ at time $t=0$ leads to non-trivial time-dependent average displacements of the probe, $\delta z(t)$, in the direction of the force. This quantity displays an intermediate-time plateau whose height depends on $F_{\text {ex }}$. This is a signature of the balance between driving force and cageing forces exerted by the host liquid. Only at long times does one observe $\delta z(t) \sim v t$, as expected for the steady-state motion. This regime allows us to determine a friction coefficient $\zeta=F_{\text {ex }} / v$. The latter displays the behavior found in earlier analyses of force-driven microrheology: close to the glass transition, it drops by orders of magnitude in a narrow force window close to a critical delocalization force $F_{\mathrm{ex}}^{\mathrm{c}}$. For smaller forces, a result close to the linear response value is obtained. At higher forces, a large-force plateau emerges.

This large-force plateau merits some discussion. Earlier work on colloidal suspensions showed it to be density dependent, with the friction coefficient $\zeta\left(F_{\mathrm{ex}} \rightarrow \infty\right)$ increasing with increasing density [7, 4]. In the MD simulations we analyze here, the large-force friction is independent on the external control parameter (temperature in our case). In our fits, this corresponds to the fact that much less weight is attributed to fluctuations perpendicular to the force. It remains to be studied how far this large-force response is indeed characteristically different in colloidal suspensions and non-colloidal thermostatted systems. Within the schematic-model analysis, no prediction on this aspect can be made.

Strikingly, a finite-velocity motion in the direction of the force also induces a diffusive MSD in the directions perpendicular to it. Extrapolating to the ideal-glass state this implies that the probe is delocalized above the force threshold not only in the prescribed direction but in all spatial directions. The data and our model suggest that the response remains almost isotropic in this sense far beyond the linear response.

The most striking feature found in the MD simulations was a super-diffusive MSD in the direction of the force at long times. Within the MCT framework for the glass-transition dynamics, at least at the level of the schematic model we are using, this finding is not explained. Following the Mori-Zwanzig projection operator formalism, MCT describes slow dynamics in terms of memory integrals; these 
furthermore can be approximated as Markovian once one considers times that far exceed the largest relaxation time appearing in the memory function. This always leads to MSD that are either arrested or diffusive as their true $t \rightarrow \infty$ asymptote. The only exception are critical points, where the memory kernels may (in the case of continuous transitions) decay as power laws, rendering in turn the MSD power-law like. Of course, outside equilibrium, this does not exclude intermediate-time super-diffusive behavior. However, within the current schematic model, we cannot reproduce the MD simulation data in this regard. It remains to be checked whether the full microscopic MCT would be able to justify the super-diffusive behavior of the MSD at intermediate times. Also, it has been argued [10] that Bouchaud's trap model can qualitatively explain the super-diffusive motion [11]; this model leads to a true non-trivial power-law asymptote for $t \rightarrow \infty$.

\section{Acknowledgments}

We thank for funding through SFB Transregio TR6 'Physics of Colloidal Dispersions in External Fields', projects A5 and A7. Th $\mathrm{V}$ thanks for funding through the HelmholtzForschungsgemeinschaft (Impuls- und Vernetzungsfonds, VH-NG-406), and through the Zukunftskolleg der Universität Konstanz.

\section{Appendix. Derivation of the MCT-MSD equations}

In this appendix, we briefly derive the equations for the MSD, starting from the microscopic MCT for force-driven microrheology presented in [4]. For notational simplicity, we use reduced units in which $k_{\mathrm{B}} T=1$ and $\sigma=1$.

The equations of motion of microscopic MCT read, for the transient probe-particle density correlation function to wavevector $\vec{q}, \phi_{\vec{q}}^{\mathrm{s}}(t)$ :

$$
\omega_{\vec{q}, \vec{q}}^{-1} \partial_{t} \phi_{\vec{q}}^{\mathrm{s}}(t)+\phi_{\vec{q}}^{\mathrm{s}}(t)+\int_{0}^{t} m_{\vec{q}}^{\mathrm{s}}\left(t-t^{\prime}\right) \partial_{t^{\prime}} \phi_{\vec{q}}^{\mathrm{s}}\left(t^{\prime}\right) \mathrm{d} t^{\prime}=0,
$$

with the memory kernel

$$
m_{\vec{q}}(t)=\int \frac{\mathrm{d}^{3} k}{48 \pi^{2} \varphi} \frac{S^{\mathrm{S}}(p)^{2}}{S(p)} \frac{(\vec{q} \cdot \vec{p}) \omega_{\vec{q}, \vec{p}}}{\omega_{\vec{q}, \vec{q}}^{2}} \phi_{p}(t) \phi_{\vec{k}}^{\mathrm{s}}(t) .
$$

Here, $\vec{p}=\vec{q}-\vec{k}$, and $p=|\vec{p}|$ have been introduced for abbreviation. The external force appears through the combination

$$
\omega_{\vec{q}, \vec{p}}=\left(\vec{q}-\mathrm{i} \vec{F}_{\mathrm{ex}}\right) \cdot \vec{p} / \zeta_{0}^{\mathrm{s}} .
$$

$S^{\mathrm{s}}(p)$ and $S(p)$ are the equilibrium static structure factors given by, respectively, the probe-host and the host-liquid interactions. They are assumed to be known from some liquid-state theory. $\zeta_{0}^{\mathrm{s}}$ is the background friction of a single free particle.

The mean-squared displacements are connected to the probe-particle density correlators in the limit $\vec{q} \rightarrow 0$. Due to the anisotropy induced by $\vec{F}_{\text {ex }}$, the spatial direction in which this limit is taken, matters. By convention, let $\vec{F}_{\text {ex }}=$ $\left(0,0, F_{\text {ex }}\right)$. We then distinguish $\vec{q}=q \vec{e}_{z}$, i.e. $\vec{q} \cdot \vec{F}_{\text {ex }} \neq 0$, where

$$
\phi_{q \vec{e}_{z}}^{\mathrm{s}}(t)=1+\mathrm{i} q \delta z(t)-\frac{1}{2} q^{2} \delta z^{2}(t)+\mathcal{O}\left(q^{3}\right),
$$

and $\vec{q} \cdot \vec{F}_{\mathrm{ex}}=0$, say, $\vec{q}=q \vec{e}_{x}$ where $\phi_{q \vec{e}_{x}}^{\mathrm{s}}(t)=1-$ $(1 / 2) q^{2} \delta x^{2}(t)+\mathcal{O}\left(q^{4}\right)$. Inserting these expressions into equation (A.1) and balancing powers of $q$, one gets equations (5) with the identifications

$$
\begin{aligned}
\hat{m}_{\perp}^{\mathrm{s}}(t) & =\lim _{q \rightarrow 0} q^{2} m_{q \vec{e}_{x}}^{\mathrm{s}}(t), \\
\hat{m}_{\|}^{\mathrm{s}}(t) & =\lim _{q \rightarrow 0}\left(-\mathrm{i} q F_{\mathrm{ex}}\right) m_{q \vec{e}_{z}}^{\mathrm{s}}(t), \\
K_{\|}^{\mathrm{s}}(t) & =\left(1 / F_{\mathrm{ex}}\right) \hat{m}_{\|}^{\mathrm{s}}(t)-\lim _{q \rightarrow 0} F_{\mathrm{ex}} \partial_{q}\left(q m_{q \vec{e}_{z}}^{\mathrm{s}}(t)\right) .
\end{aligned}
$$

Taking $\vec{q}=q \vec{e}_{x}$, we have $\omega_{\vec{q}, \vec{q}}=q^{2} / \zeta_{0}^{\mathrm{s}}$. Consequently,

$$
\begin{aligned}
\hat{m}_{\perp}^{\mathrm{s}}(t)= & \zeta_{0}^{\mathrm{s}} \int \frac{\mathrm{d}^{3} k}{48 \pi^{2} \varphi} \frac{S^{\mathrm{s}}(k)^{2}}{S(k)}\left(\vec{e}_{\perp} \cdot \vec{k}\right)^{2} \phi_{k}(t) \phi_{\vec{k}}^{\mathrm{s}}(t) \\
& +\mathrm{i} \zeta_{0}^{\mathrm{s}} F_{\mathrm{ex}} \int \frac{\mathrm{d}^{3} k}{48 \pi^{2} \varphi}\left(\vec{e}_{\|} \cdot \vec{k}\right)\left\{\frac{S^{\mathrm{s}}(k)^{2} \phi_{k}(t)}{S(k)}\right. \\
& \left.+\frac{1}{k} \partial_{k}\left(\frac{S^{\mathrm{s}}(k)^{2} \phi_{k}(t)}{S(k)}\right)\left(\vec{e}_{\perp} \cdot \vec{k}\right)^{2}\right\} \phi_{\vec{k}}^{\mathrm{s}}(t),
\end{aligned}
$$

where $\vec{e}_{\perp}$ is a unit vector orthogonal to $\vec{F}_{\text {ex }}$. The first term appearing in $\hat{m}_{\perp}^{\mathrm{s}}(t)$ is identical in its form to the one found for the equilibrium MSD; it depends implicitly on $F_{\mathrm{ex}}$ through the probe-particle correlation function. Due to the symmetry of the correlation function, $\phi_{-\vec{k}}^{\mathrm{s}}(t)=\phi_{\vec{k}}^{s *}(t)$, only the real part of $\phi_{\vec{k}}^{\mathrm{s}}(t)$ enters in this term. Likewise, only the imaginary part contributes to the second integral in $\hat{m}_{\perp}^{\mathrm{s}}(t)$. In the context of our schematic model, this contribution is neglected, assuming that the imaginary part of the correlation function remains subdominant compared to the real part.

Taking $\vec{q}=q \vec{e}_{z}=q \vec{e}_{\|}$results in

$$
\begin{aligned}
\hat{m}_{\|}^{\mathrm{s}}(t)= & \zeta_{0}^{\mathrm{s}} \int \frac{\mathrm{d}^{3} k}{48 \pi^{2} \varphi} \frac{S^{\mathrm{s}}(k)^{2}}{S(k)}\left(\vec{e}_{\|} \cdot \vec{k}\right)^{2} \phi_{k}(t) \phi_{\vec{k}}^{\mathrm{s}}(t), \\
K_{\|}^{\mathrm{s}}(t)= & \mathrm{i} \zeta_{0}^{\mathrm{s}} \int \frac{\mathrm{d}^{3} k}{48 \pi^{2} \varphi}\left\{2\left(\vec{e}_{\|} \cdot \vec{k}\right) \frac{S^{\mathrm{s}}(k)^{2}}{S(k)} \phi_{k}(t)\right. \\
& \left.+\left(\vec{e}_{\|} \cdot \vec{k}\right)^{3} \frac{1}{k} \partial_{k}\left(\frac{S^{\mathrm{s}}(k)^{2}}{S(k)} \phi_{k}(t)\right)\right\} \phi_{\vec{k}}^{\mathrm{s}}(t) .
\end{aligned}
$$

In particular, $\hat{m}_{\perp}^{\mathrm{s}}(t)$ and $\hat{m}_{\|}^{\mathrm{s}}(t)$ have the same schematic form, but due to the different scalar products with $\vec{k}$ that appear in the integrals, the weighting of the schematic correlators $\phi_{\|}^{\mathrm{s}}(t)$ and $\phi_{\perp}^{\mathrm{s}}(t)$ is taken to be different. In $K_{\|}^{\mathrm{s}}(t)$, again due to symmetry, only the imaginary part of $\phi_{\|}^{\mathrm{s}}(t)$ appears in the schematic model. Note that $K_{\|}^{\mathrm{s}}(t)$ is kept, unlike the imaginary-part contributions to $\hat{m}_{\perp}^{\mathrm{s}}(t)$ : the memory kernel $K_{\|}^{\mathrm{s}}(t)$ appears in the equations determining $\delta z^{2}(t)$ in a different convolution integral, and not as an additive correction to an existing memory kernel. 


\section{References}

[1] Squires T M and Mason T G 2010 Annu. Rev. Fluid Mech. 42 413-38

[2] Habdas P, Schaar D, Levitt A C and Weeks E R 2004 Europhys. Lett. 67 477-83

[3] Robert D, Aubertin K, Bacri J C and Wilhelm C 2012 Phys. Rev. E 85011905

[4] Gazuz I, Puertas A M, Voigtmann Th and Fuchs M 2009 Phys. Rev. Lett. 102248302

[5] Gnann M V, Gazuz I, Puertas A M, Fuchs M and Voigtmann Th 2011 Soft Matter 7 1390-6

[6] Squires T M and Brady J F 2005 Phys. Fluids 17073101

[7] Carpen I C and Brady J F 2005 J. Rheol. 49 1483-502

[8] Fiege A, Grob M and Zippelius A 2012 Granular Matter at press

[9] Zia R N and Brady J F 2010 J. Fluid Mech. 658 188-210

[10] Winter D, Horbach J, Virnau P and Binder K 2012 Phys. Rev. Lett. 108028303
[11] Bouchaud J P, Comtet A, Georges A and Le Doussal P 1990 Ann. Phys., NY 201 285-341

[12] Götze W 2009 Complex Dynamics of Glass-Forming Liquids (Oxford: Oxford University Press)

[13] Weysser F, Puertas A M, Fuchs M and Voigtmann Th 2010 Phys. Rev. E 82011504

[14] Sjögren L 1986 Phys. Rev. A 33 1254-60

[15] Gnann M V and Voigtmann Th 2012 Phys. Rev. E submitted (arXiv:1204.2407)

[16] Fuchs M, Götze W and Mayr M R 1998 Phys. Rev. E 58 3384-99

[17] Peters E A J F 2004 Europhys. Lett. 66311

[18] Zausch J, Horbach J, Laurati M, Egelhaaf S, Brader J M, Voigtmann Th and Fuchs M 2008 J. Phys.: Condens. Matter 20404210

[19] Zausch J and Horbach J 2009 Europhys. Lett. 8860001

[20] Franosch T, Fuchs M, Götze W, Mayr M R and Singh A P 1997 Phys. Rev. E 55 7153-76

[21] Krüger M, Weysser F and Voigtmann Th 2010 Phys. Rev. E 81061506 